\title{
Efficacité d'un programme basé sur l'apprentissage électronique en vue de développer quelques compétences de la traduction chez les étudiants de 4ème année à la Faculté de Pédagogie de Sohag
}

Helmi Ahmed Elwakil ${ }^{1}$, Hussein Taha Atta Salem², Adel Tawfiq Ibrahim³ ${ }^{3}$ Antar Abdelazim Abdelhamid Refaei $^{4}$

DOI:

\section{Introduction}

La méthodologie de la langue française s'évolue rapidement de nos jours pour satisfaire aux besoins de communication. Les différentes méthodologies se sont succédées, les unes qui se relient aux stratégies précédentes, les autres comme une adaptation de celles-ci aux nouvelles pistes modernes de la société.

La traduction constitue un passage important dans la construction du savoir et du savoir faire, et elle est très important de bien comprendre la situation dans laquelle on se trouve pour se faire une idée de ce qu'on va entendre ou lire. C'est pourquoi, le système universitaire égyptien prête une importance majeure à la traduction.

L'étudiant affronte une difficulté à la compréhension du texte source, mais il est capable de comprendrele texte sans difficulté quand ce texte est écrit dans la langue maternelle. Cette difficulté ne revient pas surtout d'une faiblesse de la langue étrangère, mais il semble qu'il y a une pénurie aux certaines stratègies, de la recherche documentaire dans un dictionnaire bilingue ou encore des procédés de la traduction en lisant le texte.

Le système universitaire égyptien a besoin d'une aide ou d'un soutien pédagogique capable d'intégrer les différentes stratégies pour faciliter l'acquisition de quelques compétences nécessaires. À cet égard, on doit aborder le thème de la traduction à la perspective de développer quelques compétences de la traduction et en vue de faire une ralation appropriée entre la langue maternnelle de l'apprenant et la pratique de la langue étrangère. Aussi la traduction joue un rôle essentiel dans le rapprochement des peuples en invitant à d'autres enquêtes sociologiques et d'autres recherches scientifiques.

Pratiquer la traduction en cours de langue ne signifie pas un retour aux méthodes classiques d'apprentissage ou bien sûr d'enseigner sa théorie, mais plutôt à utiliser la traduction comme un outil pour manipuler cet apprentissage à des fins pédagogiques; cela suscite un développement des capacités intellectuelles et culturelles des apprenants, 
parallèlement à l'acquisition des compétences linguistiques.

Vinay et Darbelnet, parmi les premiers, qui ont convaincu le monde universitaire de la nécessité d'enseigner la traduction selon une méthode rigoureuse, mieux adaptée à la société et à ses besoins actuels, et fondée sur des principes qui, quoique inspirés par la linguistique, sont applicables à l'enseignement des langues vivantes dans la perspective de traduction. (Vinay et Darbelnet, 1965, p.49)

Tenant compte du fait qu'on veut faire un passage de la théorie de traduction à la pratique, on met l'accent sur la théorie comme une base de formation traductionnelle, c'est-à-dire on trouve des procédures pertinentes pour appliquer une méthode bien déterminée.

Comme l'a dit Aubin: "la théorie soutient la pratique et n'apparaît le plus souvent que dans ses résultats dans les programmes de formation permettant aux étudiants d'accélérer leur acquisition des compétences essentielles à la pratique'. Autrement dit, si la théorie n'existait pas, il faudrait l'inventer car aucun programme de formation ne serait viable sans une réflexion théorique sur la pratique du métier." (Aubin, 2003, p. 438)

D'après ce point de vue, Aubin met l'accent sur l'importance de la théorie à développer les compétences de la traduction. À cet égard, la théorie joue un rôle majeur dans la contextualisation de l'apprentissage de la traduction.

[L'évolution de la traductologie correspond d'ailleurs à l'intégration de variables toujours plus nombreuses pour analyser l'activité traduisante. Dans ce contexte, l'étude de Valentine intitulée: "Traductologie, traduction et formation: vers une modalisation de la formation en traduction - l'expérience canadienne.", prend comme point de départ l'examen du concept de formation, expose les enjeux et les finalités de celle-ci ainsi que les composantes et les centrations qu'on y décèle. Cette étude s'attache à examiner les théories de la traduction dans le but de déterminer la place que réservent celles-ci à la formation (Valentine, 1996, p.27).

En effet, ce processus s'appuie autant sur différentes compétences, et on peut les répartir selon leurs étapes de la pratique en plusieurs compétences constitutives. Il y a des compétences qu'on offre au début des cours aux étudiants avant de traduire, c'est-a-dire, les compétences prétraductionnelles,les compétences primordiales avant de traduire ainsi que les compétences concernant le vocabulaire, la grammaire, la syntaxe, la ponctuation, l'analyse, la rédaction, la reformulation, la déverbalisation, le style... etc. On trouve aussi des compétences durant le travail, c'est-à-dire des compétences directement 
liées à l'acte de traduire comme la compétence de documentation, la compétence de révision d'un texte, et de l'enrichissement de la culture générale de la recherche. Par suite les autres compétences; ce sont celles de la pratique.

L'étude de Léchauguette a montré que dans ce secteur, la réflexion traductive qui porte sur le texte dans sa mise en page. L'unité de traduction s'hybride, puisqu'elle est composée de rubriques textuelles aux fonctions communicatives précises et d'éléments visuels.

L'étude de Forget a montré que les dictionnaires électroniques offrent certes des avantages en regard des versions papier: consultation flexible, manipulation aisée, navigation rapide, capacités de recherche puissantes et variées, fonctions bureautiques pratiques. Cette étude indique l'utilité du dictionnaire électronique dans la traduction. Par exemple, la pésentation $\mathrm{du}$ dictionnaire électronique diffère de celle du dictionnaire papier, comme la mise en page (Forget, 1999, p.18).

Pour améliorer les compétences de la traduction, on essaie d'abord d'améliorer les sous-compétences (les compétences secondaires) comme la compétence linguistique, culturelle, terminologique, thématique, professionnelle, textuelle, documentaires, communicative ...etc. De ce sens l'étude de Solhy intitulée: " Un programme suggéré pour développer quelques compétences principales de la traduction chez les professeurs de l'anglais et son effet sur leurs attitudes vers la traduction."(Solhy, 2006, P.112),affirme qu'il y a quatre compétences principales de la traduction: la compétence de la compréhension écrite, la compétence documentaire, la compétence analytique, et la compétence de la recherche (comment utiliser les dictionnaires bilingues, les encyclopédies, pour chercher des expressions scientifiques spécialisées).

Ainsi, pour acquérir ces compétences on doit enrichir le bagage linguistique de l'apprenant pour comprendre ce qu'on traduit et pour voir comment améliorer les compétences actuelles, avant d'acquérir les compétences manquantes chez ces apprenants. Pour faire cette amélioration on a besoin de pratiquer quelques procédés d'équivalence et d'adaptation.

De ce qui précède, il ressort que l'acte de la traduction relève d'une compétence intellectuelle reposant sur une bonne connaissance de deux langues en jeu. Il y a une tentative spécifique de s'interroger sur la place à accorder au savoir-faire par rapport au savoirtraduire.

On tente à déterminer les compétences principales qui doivent être traitées dans n'importe quel programme de formation à la traduction.Cependant, il paraît raisonnable de vérifier le niveau de traduction d'après ces 
critères d'évaluation afin de mesurer les résultats attendus de la traduction à travers de nouvelles activités diversifiées, et des critères qui s'accordent avec notre société.

Pour ce faire, on s'intéresse à élaborer des techniques multiples pour améliorer quelques compétences de la traduction à l'aide de l'apprentissage électronique car cet apprentissage s'est imposé comme une nouvelle approche de la formation continue des [TIC] sous une forme du e-learning comme : (naviguer sur quelques sites d'Internet concernant la traduction, démontrer le logiciel, mettre en ligne, télécharger des plates-formes de la traduction, naviger dans un système de traduction assisté par l'ordinateur, etc .... .)

Ainsi, on appliquera quelques modes modernes d'apprentissage à l'aide du e-learning comme la base des données de partage (discussions), les logiciels spécifiques de traduction en ligne, les forums, les dictionnaires électroniques, ... etc. De plus, on essaie d'appliquer d'autres méthodes avec les apprenants pour bien évaluer leur travail en s'appuyant sur la stratègie de l'apprentissage électronique.

On essaie d'élaborer des stratégies prometteuses pour favoriser l'usage et l'intégration pédagogique de la traduction. C'est pourquoi, ici le chercheur s'intéresse à manifester le rôle primordial de l'apprentissage électronique, afin que les étudiants acquièrent quelques compétences de la traduction et rectifient leurs erreurs, ajouter, et reformuler.

Parmi les pédagogues qui se sont préoccupés par l'utilisation de l'informatique à la traduction, l'étude de Catherine Arrouart: "Cadre d'acquisition de compétences en informatique dans la formation universitaire en traduction."Cette étude affirme que il y a une nécessité de posséder les compétences en informatique pour pratiquer des activités traductionnelles (Arrouart, 2003, p. 19).

De même, Gouadec réaffirme ce besoin actuel de proposer des formations complémentaires aux traducteurs, dont le programme serait l'analyse des produits ou matériaux à localiser, la mise à point des techniques de traduction de ces matériaux et l'acquisition des compétences technologiques inhérentes à la localisation. Gouadec, affirme que la familiarisation avec les outils informatiques et l'Internet serait indispensable dans toute formation à la localisation (Gouadec, 2002, p. 23).

Ces études montrent que les étudiants actuellement ont besoin d'expérimenter et d'assimiler des méthodes efficaces pour accéder rapidement à l'information. À l'aide de la technologie du e-learning comme une piste numérique très moderne, ils peuvent développer les compétences nécessaires à la traduction. 


\subsection{Problématique de l'étude:}

Étant donné, qu'il y a une grande importance des compétences de la traduction, dans l'acte de traduire, les apprenants doivent acquérir ces compétences pour faciliter leur tâche et pour réexprimer en langue cible le sens du message d'une manière compréhensible.

La cause principale de ce problème est due au manque de quelques notions linguistiques chez eux aux deux langues (source et cible). Ces apprenants sont incapables de faire acquérir ces compétences primordiales à leurs futurs étudiants. On constate que l'étudiant égyptien après avoir terminé ses études à l'université, se montre incapable de bien traduire un texte et aussi de perfectionner le travail de la traduction de thème et de version.

Pour ce faire, on a effectué quelques rencontres avec certains porfesseurs dans les Facultés de Pédagogie. Ils ont tous affirmé que le niveau des étudiants était très faible du côté de la traduction et ces étudiants avaient besoin de cours de traduction plus professionnels.

De plus, on a appliqué une étude-pilote au cours de l'année scolaire 2011/2012 auprès des étudiants de la 4éme année à la Faculté de Pédagogie, Université de Sohag dont le nombre atteint 30. Cette étude-pilote s'est composée d'un test de traduction ayant trois questions dans le but de vérifier le niveau de la traduction et les attitudes envers la traduction chez ces apprenants. Chaque question se compose de quelques items visant à vérifier le niveau des compétences de la traduction chez ces apprenants.

L'étude-pilote offre l'avantage de fournir des renseignements utiles pour éclaircir la faisabilité de l'étude proposée. Ces renseignements sont complétés par des données que l'on obtient au moyen d'une grille synoptique. Cette grille a examiné les questions internes liées au test de traduction. On a calculé la moyenne des notes comme suit:

On a calculé la moyenne des notes, la valeur la plus haute $35.7 \%$; la valeur la plus basse $12 \%$. La présentation de ces proportions montre que chaque item ne suffit pas à définir les compétences de la traduction chez ces apprenants. Ainsi, les résultats obtenus nous ont alarmés sur des lacunes esssentielles, comme telles:

- La plupart des apprenants utilisent la traduction directe ou littérale (mot à mot) pour traduire les textes déterminés, et ils sont incapables d'adapter quelques termes ou idées de la langue source à la langue cible.

- La majorité des apprenants recourrent à l'emprunt de groupes de mots comme unités séparées sans aucune équivalence.

- Certains étudiants ne respectent pas la fidélité du texte, c'est-à-dire, ils ne peuvent être 
fidèles à l'auteur, à la réalité ou au caractère interne du texte.

Ces proportions donnent l'occasion de constater que les étudiants de l'étude-pilote éprouvent une faiblesse évidente dans l'acquisition de quelques compétences de la traduction.
On peut donc mentionner que la problématique de la recherche réside en «lafaiblesse des compétences de la traduction en FLE chez les futurs enseignants des départements de français dans les Facultés de Pédagogie ».
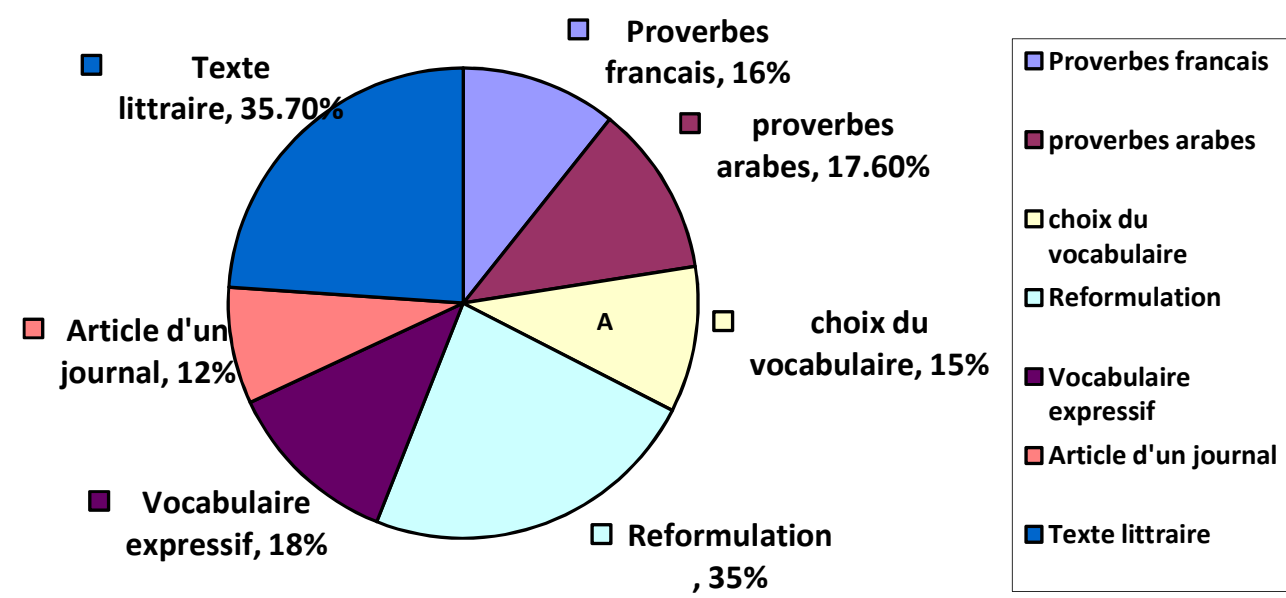

Diagramme N1 : Résultats de l'étude-pilote

L'étude actuelle rejoint ces préoccupations en essayant de répondre à la question suivante:

Quelle est l'efficacité de l'apprentissage électronique pour développer les compétences de la traduction en FLE chez les étudiants de la 4ème année du département de français dans la Faculté de Pédagogie de Sohag?

\subsection{Objectifs de l'étude:}

Cette étude vise à développer quelques compétences de la traduction chez les étudiants de la $4^{\text {éme }}$ année, département defrançais, Faculté de Pédagogie de Sohag.

\subsection{Limites de l'étude:}

Cette étude se limite à:
1. un échantillon d'étudiants de la $4^{\text {éme }}$ année, section de français, Faculté de Pédagogie de Sohag qui étudient la langue française comme deuxième langue étrangère. Ainsi, le nombre de ces étudiants atteint 21 au total de 31. Pour réaliser les analyses, on a retenu certaines caractéristiques de ces apprenants; les données relatives à l'âge, au genre, et au parcours scolaire, comme suit:

- Ils ont les mêmes circonstances, c'est à dire qu'ils appartiennent à la 4ème année, Faculté de Pédagogie, Université de Sohag. 
- L'échantillon comprend 5 étudiants et 16 étudiantes.

- Ils ont 21 ans.

- Ils sontétudiants en 4ême année, section de français. Ces étudiants seront licenciés et deviendront enseignants de français.

- Tel échantillon comporte un seul groupe qui étudie le programme proposé.

- Ces étudiantes ont besoin de développer leurscompétences traductionnelles.

2. développer les cinq sous-compétences de la traduction: linguistique, culturelle, communicative, de transfert interlinguistque et terminologique.

\subsection{Importance de l'étude:}

L'importance de cette étude se manifeste aussi sur deux plans:

\section{a-Sur le plan théorique:}

- Le cadre théorique de notre étude devrait permettre d'augmenter lesconnaissances et les techniques modernes en matière de l'enseignement de la traduction en FLE ainsi qu'en matière de la pratique de l'apprentissage électronique comme une stratégie efficace pour élaborer un chemin aménagé pour l'apprentissage d'une langue étrangère.

- Contribuer à surmonter les difficultés rencontrées en traduisant un texte ou une activité par exemple.
- Aider à développer le niveau des apprenants de la 4éme année desfacultés de pédagogie à trouver plusieurs outils et solutionspour bien traduire, ce qui augmente aussi la qualité de traduction.

- Élaborer une liste de toutes les compétences de la traduction nécessaires aux apprenants de la 4éme année des facultés de pédagogie. Cette liste peut être une bonne référence pour beaucoup d'autres recherches dans ce domaine.

- Présenter un modèle afin d'élaborer un siteInternet concernant le domaine de la traduction.

- Élaborer unegrille d'évaluation en vue de mesurer les résultats attendus des apprenants concernant les compétences nécessaires de la traduction. Cettegrille aide les autres chercheurs à analyser et à évaluer le travail de la traduction.

\section{b-Sur le plan pratique:}

- Le programme proposé peut être appliqué dans les différentes facultés de pédagogie.

- Les instruments que le chercheur élabore pour évaluer les compétences de la traduction peuvent servir comme des données pédagogiques dans d'autres recherches.

\subsection{Hypothèses de la recherche:}

L'étude actuelle vise examiner l'hypothèses suivante: "il existe une différence significative 
entre les moyennes des notes des étudiants de l'échantillon au test de la traduction avant et après l'application du programme en faveur $\mathrm{du}$ post tes".

\subsection{Outils de la recherche:}

1- Un pré-post test de traduction.

2- Un programme basé sur l'apprentissage électronique.

\subsection{Méthodologie du programme :}

Dans cette étude, le chercheur a utilisé deux méthodes:

1- la méthode de la traduction pédagogique:

La méthode de la traduction pédagogique comporte les exercices de thème et de version. On adopte la méthode de (Vinay et Darbelnet, 1958), cette méthode se basesurla traduction pédagogique pour permettre aux apprenants d'expérimenter les différentes méthodes en traduction. Pour la traduction pédagogique, il s'agissait de phrases décontextualisées à traduire en employant les procédés et les techniques de traduction tels que décrits par Vinay et Darbelnet dans la stylistique comparée du français et de l'anglais. Nous avons donc adopté la méthode Vinay et Darbelnet, pour traduire des textes appartenant au domaine de l'apprentissage en ligne et des TIC à l'aide d'outils interactifs afin que l'apprenant puisse contextualiser son apprentissage en traduction.

\section{2- la méthode quasi-expérimentale:}

Il s'agit de mesurer l'effet de la variable indépendante sur la variable dépendante, ainsi que pour calculer la différence entre la moyenne des notes des apprenants au test de traduction avant et après l'utilisation de l'apprentissage électronique.

\section{8. Étude théorique}

L'apprentissage électronique affronte plusieurs défis économiques, technologiques et sociaux, mais avant de parler de ces défis, on doit mettre l'accent sur la situation du contenu didactique d'apprentissage de la traduction sur Internet. Pour faire évaluer l'apprentissage électronique en traduction, on doit mettre à disposition des outils spécifiques sur l'Internet; ceci aide à acquérir quelques compétences de la traduction

Le rôle des sites didactiques augmente à travers la gestion numérique de la traduction. Alors, le chercheur va traiter dans cette partie de ce chapitre quelques outils qu'il va pratiquer dans son programme, comme l'utilisation du Web, des logiciels spécifiques en traduction. Il va mettre l'accent sur ces outils, en montrant leur importance dans la phase de l'acquisition des compétences de la traduction. 


\subsubsection{Les compétences de la traduction et les outils informatiques}

Les outils informatiques d'aide à la traduction font de plus en plus partie de l'environnement professionnel de l'apprenti-traducteur. Ils désignent différents types de logiciels de traduction assistée par ordinateur ou mémoires de traduction, base de données linguistiques qui enregistre la traduction au fur et à mesure afin de la réutiliser ultérieurement, des dictionnaires électroniques pour la recherche immédiate des informations requises, des forums ... etc. Ces outils ont plusieurs avantages comme suit:

1- Traduction rapide de qualité sans utiliser 2 fois la même phrase ;

2- Gestion terminologique: acronymes, synonymes et abréviations qui permettent de définir les règles d'usage pour chacun des termes;

3- Traduction précise et efficace permettant une amélioration de la précision de la cohérence, et de la qualité de la traduction;

4- Recherche spécifique du vocabulaire, des expressions, des tournures, et des passages textuels;

Ainsi, toute formation en traduction comprend une pratique aux outils de TICE et aux programmes d'aide à la traduction. Le choix des outils à intégrer dans l'enseignement s'avère fondamental et reflète la réalité de besoin de

cette formation; ce choix nécessite de confronter les apprenti- traducteurs à des outils variés et à les doter des capacités d'adaptation qu'exige l'utilisation des outils présents dans l'exécution des tâches, et dans l'environnement professionnel.Le choix doit également tenir compte de l'évolution des outils disponibles sur le marché.

Alors, l'apprenant doit maîtriser l'art d'écrire dans sa langue au même degré que la langue source, posséder des capacités d'analyse et de synthèse; il doit également être familiarisé avec les sujets et les textes spécialisés.Les compétences qu'il va acquérir doivent porter sur un ensemble d'habiletés, de connaissances informatiques et de motivations qui pourraient garantir l'efficacité de l'activité pratiquée.

Il est vrai que l'apprenant ne peut pas se passer des outils traditionnels tels les ouvrages lexicographiques, les banques de données, l'internet etc...tout en utilisant des outils et des logiciels propres à la traduction telle la traduction à l'aide du e-learning, la traduction assistée par ordinateur qui l'aide dans l'expression orale et écrite et lui assure des habiletés linguistiques.

Hurtado $(2008,28)$ affirme que la compétence instrumentale est primordiale pour bien traduire, et elle concerne la capacité d'utiliser les outils, de coopérer et d'argumenter.Elle peut être de 
type cognitif, méthodologique, technologique et linguistique.

La compétence disciplinaire est, elle aussi, essentielle et permet à l'apprenant de maitriser l'interprétation des textes spécialisés en puisant dans la sous-compétence extralinguistique, la connaissance biculturelle encyclopédique et thématique. Bordeur, 2008 affirme qu'il y a trois types essentiels de compétences. La figure suivante d'après Bordeur montre la différence entre ces compétences.

\begin{tabular}{|c|c|c|}
\hline $\begin{array}{l}\text { Compétences } \\
\text { Instrumentales }\end{array}$ & $\begin{array}{c}\text { Compétences } \\
\text { Interpersonnelles }\end{array}$ & $\begin{array}{l}\text { Compétences } \\
\text { Systémiques }\end{array}$ \\
\hline $\begin{array}{l}\text { - Capacité d'analyse et de } \\
\text { synthèse } \\
\text { - Capacité d'organisation et de } \\
\text { Planification } \\
\text { - Connaissance générale de } \\
\text { base } \\
\text { - Approfondissement de la } \\
\text { connaissance de base de la } \\
\text { profession } \\
\text { - Communication orale et } \\
\text { écrite en langue maternelle } \\
\text { - Connaissance d'une langue } \\
\text { seconde } \\
\text { - Habiletés informatiques de } \\
\text { base } \\
\text { - Habiletés de gestion de } \\
\text { l'information } \\
\text { - Résolution de problems } \\
\text { - Prise de décisions }\end{array}$ & $\begin{array}{ll}\text { - } & \text { capacité de critique et } \\
& \text { d'autocritique } \\
\text { - } & \text { travail en équipe } \\
\text { - } & \text { habiletés } \\
& \text { interpersonnelles } \\
\text { - } & \text { capacité de travail } \\
& \text { dans une équipe } \\
& \text { interdisciplinaire } \\
\text { - } & \text { capacité de } \\
& \text { communiquer avec des } \\
& \text { experts d'autres } \\
\text { - } & \text { Disciplines } \\
\text { - } & \text { appréciation de la } \\
& \text { diversité et de la } \\
& \text { multiculturalité } \\
\text { - } & \text { capacité de travailler } \\
\text { - } & \text { dans un context } \\
& \text { international } \\
\text { - } & \text { comportement Ethique }\end{array}$ & 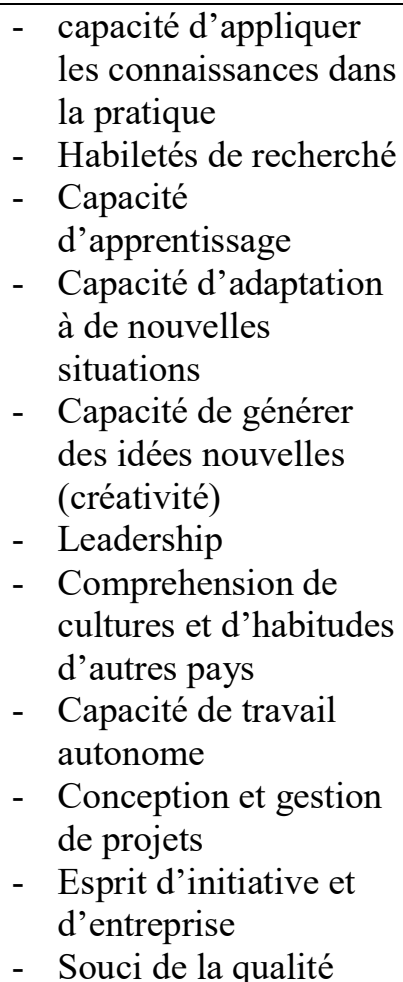 \\
\hline
\end{tabular}

Figure 1: Compétences générales d'après le projet Tuning

Selon Bordeur, Les competences interpersonnelles comprennent des habiletés d'interaction avec autrui; elles favorisent les processus d'interaction sociale et la communication. Les compétences systémiques représentent une combinaison de compréhension, de sensibilité et de connaissances pour permettre à l'individu de comprendre comment les parties d'un tout sont reliées entre elles. (Brodeur, 2008, p.24\&25)

Les compétences ainsi requises dans le processus d'apprentissage de la traduction (compétence interprétative, compétence de coordination des efforts, compétence de transfert, compétence de base en gestion) exigent une combinaison des outils du e- 
learning pour atteindre ses objectifs et pour aboutir à la traduction de qualité.

\subsubsection{L'utilisation du Web en traduction}

Le web offre un accès à une quantité considérable de documents électroniques dans plusieurs domaines, surtout le domaine de la traduction. Il y a des sources multiples des informations concernant la traduction, comme les sources textuelles, encyclopédiques, terminologiques, et les dictionnaires électroniques bilingues. En cherchant ce qu'on veut connaitre, il suffit de préciser des mots clés, avec d'autres indications concernant un texte ou un document écrit à traduire.

Le nombre des sites d'Internet ayant le but de traduire, augmentent rapidement, surtout la traduction de célèbres logiciels en ligne comme le moteur de recherche Google et Babylon.

Pourtant, on constate que ces sites sont classiques, et qu'on trouve un certain nombre de faiblesses méthodologiques dans l'emploi de ces outils. Mais le développement du Web, aide le traducteur à chercher, à négocier, à synchroniser, à partager les informations nécessaires pour traduire un texte. Comme le souligne Gouadec (2002, p.15), le développement du Web constitue une véritable révolution. Il modifie considérablement les possibilités et la manière de conduire une recherche d'informations. Le foisonnement de sites dans de nombreuses spécialités et dans de nombreuses langues permet maintenant au traducteur d'accéder instantanément à une énorme quantité d'informations.

Les sites du Web permettent la consultation d'autres traducteurs, des locuteurs natifs de la langue d'arrivée spécialistes du domaine. La recherche sur le Web aboutit à plusieurs solutions possibles. En effet, il est possible d'emprunter une énorme quantité des pages contenant des textes et des hypertextes. L'apprentissage à l'aide du Web, est un mode du e-learning.

Alors, la transmission du savoir connait une accélération et une extension sans précédent, qui abolit l'espace et le temps. La rapidité de l'information ne supprime ni la lenteur de la formation traductionnelle, ni l'influence sur l'information de son origine- socio-culturelle. Avec les TIC, l'enseignant sera en mesure de contextualiser l'information. La transmission présuppose la présence d'un enseignant technique qui surveille l'arrivée des documents à traduire, et en fait la distribution dans les cabines. Cet enseignant assure la liaison à distance avec les apprenants, il intervient également pour aider et guider ces apprenants à bien accomplir leur tâche. 


\subsubsection{Des logiciels de traduction:}

La traduction intervient à deux endroits précis dans la communication multilingue, soit à la langue source, soit à la langue d'arrivée. Mathieu Guidère (2008) indique que le processus de traduction repose sur un large éventail de connaissances, parmi lesquelles figurent en première place les connaissances grammaticales de la langue source et de la langue cible. Il est clair que ces connaissances linguistiques sont insuffisantes pour assurer une traduction correcte et de bon niveau. (Guidère, 2008, p.60)

Pour cela, on a besoin d'envisager la pratique des applications informatiques relativement importantes pour améliorer la qualité de la traduction. Les logiciels d'Internet est l'une de ces outils informatiques qui permettent au traducteur de construire sa propre banque d'informations. Dans ces logiciels, le traducteur obtient des outils et des liens variés, exhaustifs, adéquats et fiables. Il peut faire une recherche terminologique consacrée à la traduction dans les domaines techniques, scientifiques, et juridiques, ... etc.

Deux types de logiciels sont actuellement disponibles pour l'étude des mots en contexte: ceux qui ont été conçus pour le traitement en lot et ceux destinés à une utilisation en mode conversationnel. Le premier type de logiciels, employé depuis une quarantaine d'années,

permet de produire des index et des concordances sur papier. Le deuxième type, plus récent, autorise une exploration des contextes à partir d'un dispositif visuel, d'un écran cathodique ; cet instrument de travail d'un type nouveau est beaucoup plus souple et offre de nouvelles possibilités aux chercheurs. (Guidère, 2008, p.25)

Honig, (1991:78), affirme qu'on évalue la qualité des logiciels en fonction de trois critères:

1- Qualité du son : Il s'agit de la clarté des voix du logiciel. Nous cherchons à évaluer la qualité des commentaires contenus dans les logiciels: l'intonation varie-t-elle en fonction du sujet abordé ?

2- Qualité de la lecture: Il s'agit de la qualité des dessins et des activités interactives proposées par le logiciel. Quelle est la qualité des textes proposés sur les fenêtres de l'écran? La qualité des images, des animations, est-elle satisfaisante ?

3- Temps utilisé par sujet: Il s'agit de la durée de projection de chaque écran et du commentaire qui en est donné.

La pratique de chaque logiciel a besoin d'un soutien pédagogique concernant la qualité de son et des médias audiovisuels déposées, la qualité de la lecture concernant le type des liens interactifs. Ce processus ne nécessite pas un temps de projection trop long et des explications 
trop nombreuses. Chaque écran doit être clair et facilement compréhensible par les apprenants.

Ainsi, les logiciels jouent un rôle important dans le processus de l'acquisition de compétences de la traduction. À partir d'un texte enregistré sur un lien informatique, ou sur une icône, l'ordinateur découpe le texte en mots. Un mot y est défini comme toute suite de caractères comprise entre deux espaces blancs, deux signes de ponctuation ou un espace blanc et un signe de ponctuation.

\section{9. Étude quasi-expérimentale}

Outils de la recherche, procédures, et application de ces outils.

\subsubsection{Un près/post test de traduction}

\subsubsection{Les objectifs du test:}

Ce test a pour objectif d'évaluer les niveaux des apprenants concernant les compétences de la traductionexactement chez les apprenants de la quatrième année section de français, Faculté de Pédagogie de Sohag.

\subsubsection{Contenu du test.}

Le test écrit se compose de trois textes ( 2 textes de version, 1 texte de thème), suivis des questions qui varient entre:

1. Répondez aux questions suivantes d'après le texte.

2. Choisissez le bon groupe d'après le texte.

3. Mettez vrai ou faux d'après le document.
4. Recherchez les éléments lexicaux qualifiant le thème du texte.

5. Complétez les phrases par les mots suivants.

6. Trouvez l'équivalent arabe ou français de ces phrases.

7. Traduisez les expressions idiomatiques suivantes.

8. Traduisez les proverbes suivants en français.

9. Traduisez le texte ci-dessus en arabe.

10. Traduisez le texte ci-dessus en français.

\subsubsection{Passation du test.}

Nous avons choisi 21 apprenants de la quatrième année département de français, faculté de Pédagogie de Sohag.

A savoir que l'application de ce test pendant l'étude pilote nous a permis de calculer la durée de l'application du test, sa fidélité et sa validité.

\section{Durée du test:}

Pour déterminer la durée convenable pour répondre aux deux volets du pré-test nous avons effectué une étude pilote. Nous avons demandé à un échantillon d'apprenants (8 apprenants) de répondre au test de traduction.

Nous avons calculé la durée prise par l'apprenant qui a fini en premier, et celle prise par l'étudiante qui a terminé son travail en dernier, pour trouver la durée moyenne de répondre au test .Ainsi nous avons trouvé que la durée globale de l'application du pré-test est de 2 heures 45 minutes comme suit: 


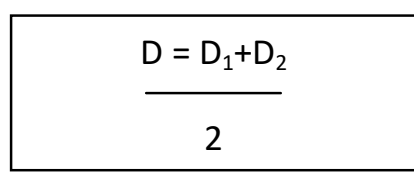

La moyenne de temps $=$ temps pris par le premier + temps pris par le dernier

La moyenne de temps $=150+180=165=2$ heures 45 minutes

\subsubsection{Fidélité du test:}

Pour calculer la fidélité du test, nous avons appliqué ce test sur 8 apprenants au mois de septembre 2015, donc au début du semestre; puis nous l'avons réappliqué une semaine après pour comparer les résultats des apprenants dans les deux fois.

\begin{tabular}{l|l}
\multicolumn{2}{c}{ Tableau $\mathbf{~ N}^{\mathbf{1}}$} \\
\hline N of Items & Cronbach's Alpha \\
\hline 27 & 0.776
\end{tabular}

Nous avons calculé le coefficient de fidélité du test de la traduction en utilisant le programme statistique SPSS, et d'après l'équation «Alpha Cronbach's», le coefficient de fidélité $=0.77$, c'est-à-dire le test est valable, donc il est applicable.

\section{La validité du test:}

Pour mesurer la validité du test de traduction, nous avons calculé les coefficients de simplicité et de difficulté de corrélation entre les notes des apprenants avant et après l'application du test de
D: la durée. $\mathbf{D}_{\mathbf{1}}$ : durée prise par l'apprenant qui a fini en premier. $\mathbf{D}_{2}$ : durée prise par l'apprenant qui a fini en dernier.

la traduction en utilisant le programme statistique SPSS.

\subsubsection{Le programme de l'étude}

Ce programme ayant pour but de développer quelques compétences de la traduction et l'attitude envers le e-learning chez les étudiants de la 4ème année section de français à la Faculté de Pédagogie de Sohag. Pour arriver à la forme finale du programme, le chercheur a consulté les études antérieures dans le domaine de didactique de FLE, aussi les études précédentes dans le domaine du e-learning des langues étrangères.

\subsubsection{Contenu du programme:}

Le programme de cette étude comprend (5) unités avec une introduction théorique qui est présentée sous forme d'une unité ayant pour but de donner aux apprenants une idée approfondie des stratégies d'apprentissages en général et des stratégies du e-learning choisies en particulier en leur montrant l'intervention et l'effet du elearning sur l'acquisition des compétences de la traduction. 
1. Première unité : Les différents aspects de la traduction.

Cette unité est une pratique à la traduction bilingue.Ona pu constater que les apprenantsont suivi un cours de traduction dans des combinaisons linguistiques différentes mettanten jeu leur langue maternelle pour améliorer leur compétence linguistique. Combinaison culturelle.

2. Deuxième unité:Cette unité ayant pour but de faire une adaptation culturelle, il y a des textes et des exercices variés à traduire touchant le côté de la culture de deux langues.

3. Troisième unité: Communication traductologique médiatisée.

Cette unité comporte quelques activités pour faire comprendre que la composante linguistique d'un texte renvoie simplement à la connaissance de sa langue.Les prérequis sont la compréhension de l'arabe et la maîtrise du français.

4. Quatrième unité: Le transfert inter linguistique.

Cette unité ayant pour but de réaliser une traduction collective où les apprenants repartis en groupes de travailpour partager et transformer les informations.

5. Cinquième unité: $\mathrm{La}$ recherche terminologique et thématique.
La cinquième unité concerne la réalisation d'une recherche sur une thématique appartenant au domaine des technologies éducatives et de l'apprentissage en ligne en traduction.

\subsubsection{La pratique des activités}

L'étape des activités du programme représente le noyau de notre travail pour acquérir les compétences de la traduction, et pour développer l'attitude des apprenants envers l'apprentissage de la traduction. Ce sont les étapes mentionnées préalablement:

- La compréhension globale du texte.

- La construction du sens global du texte.

- La reformulation du texte.

- La synchronisation du texte avec un tuteur.

Dans la catégorie "Activités", à la page d'accueil concernant les unités du programme, il y a des liens pour faire les exercices, des liens pour déposer des documents word $\mathrm{du}$ programme concernant les exercices du programme et les activités sous forme word tout en ayant recours au forum, l'apprenant peut créer des parcours, des évaluations, proposer des liens, créer un glossaire, éditer un nouveau message, émettre ses appréciations et ses considerations.

Il peut cliquer ensuite sur le lien (poursuivre pour voir l'autre activité). Le chercheur alimente son cours par des types d'exercices variés 
figurant sur les activités du programme. Chaque compétence a ses propres activités.

Dans ce cadre, lechercheur peut gérer les groupes d'apprenants, utiliser les outils de partage, d'accompagnement, et de communication, gérer les agendas et l'espace travaux de textes à traduire. Il pourrait aussi donner des notes, des conseilsà l'aide du "Forum", "discussions"... afin que les apprenants puissent produire un texte cible.

\subsubsection{L'évaluation:}

L'apprenant exerce un travail d'auto évaluation en cliquant sur le lien "auto-évaluation". Dans la partie centrale de l'écran, il clique sur l'image pour vous inscrire et tester à l'aide de ce lien. Après avoir testé, il y a une auto correction immédiate de ce faire.

Par la suite, ilpeut, immédiatementcréer un feedback en cliquant sur le bouton associé, ilpeut aussi reconnaitreses fautes: Cette technique lui permet d'améliorer sa compétence linguistique de la traduction.

L'écran de l'icône auto-évaluation se compose de plusieurs formes de variations: tests d'autoévaluation, auto correction, tests d'unité, fiche d'observation et d'auto-évaluation. En cliquant sur l'icône, “ajouter une remarque”, l'apprenant peut alimenter le cours par des remarques qu'il voit précieuses.

\subsection{Résultats du pré/post test}

L'analyse porte sur

- Les progrès réalisés entre le pré- test et le post- test.

- Efficacité d'un programme basé sur l'apprentissage électronique pour développer quelques compétences de la traduction et l'attitude envers son apprentissage chez l'échantillon.

On a analysé les résultats obtenus après la passation du pré/post test de la traduction.

Pour l'analyse statistique des résultats, le chercheur a utilisé le programme statistique SPSS pour calculer la moyenne arithmétique des notes, l'écart type et la valeur de $\mathrm{T}$.

Les opérations statistiques utilisées dans la recherche sont les suivants:

1) "T test": Utilisé dans la comparaison entre les moyennes séparées comme les notes des étudiants de groupes expérimentaux et témoin.

2) "Corrélation" Indique l'intensité et la direction de la relation entre deux variables.A côté des moyennes statistiques et les écarts types des notes des apprenants.

Pour achever les opérations statistiques cidessus, à travers le logiciel statistique SPSS (Statistical package of social science), on va expliquer en détail l'opération convenable à chaque hypothèse comme suit:Pour l'hypothèse $\mathrm{N}^{\circ} 1$ «Il existe une différence significative entre 
les moyennes des notes des étudiants de l'échantillon au test de la traduction avant et après l'application du programme en faveur du post test».
On a calculé la valeur de "T test" pour comparer les moyennes des notes des apprenants au pré/ post test d'acquisition pour savoir la différence entre les notes avant et après l'application des activités proposées, commele tableau suivant l'indique:

Tableau $\mathbf{N}^{0} 3$

\begin{tabular}{|c|c|c|c|c|c|c|c|c|}
\hline \multirow[b]{2}{*}{ Données } & \multirow[b]{2}{*}{$\mathrm{n}$} & \multicolumn{2}{|l|}{ Pré } & \multicolumn{2}{|l|}{ Post } & \multirow[b]{2}{*}{ "t" } & \multirow[b]{2}{*}{ df } & \multirow[b]{2}{*}{$\mathrm{p}$-valeur } \\
\hline & & $\mathrm{Ma}$ & $\begin{array}{l}\text { Std. } \\
\text { Déviation }\end{array}$ & $\mathrm{Ma}$ & $\begin{array}{l}\text { Std. } \\
\text { Déviation }\end{array}$ & & & \\
\hline $\begin{array}{l}\text { Pré et post } \\
\text { test de la } \\
\text { traduction }\end{array}$ & 21 & 20.43 & 3.85 & 38.50 & 5.11 & 11.89 & 20 & 0.000 \\
\hline
\end{tabular}

$\mathbf{N}=$ nombre d'étudiants. $\mathbf{M a}=$ moyenne arithmétique. Std Déviation = la ligne des fréquences (l'écart-type). $\mathbf{D f}=$ degré de liberté. $\mathbf{T}=$ valeur de $(\mathrm{t})$ test. $\mathbf{P}$-valeur $=$ valeur de l'influence .

D'après le tableau précédent, la moyenne des traduction avant et après l'application du notes des apprenants au pré test était 20.43 et programme en faveur du post test.

l'écart type 3.85 , mais au post test la moyenne des notes des apprenants était 38.50 et l'écart type 5.11. La valeur de «T test» était 11.89 avec une p-valeur 0.000. Donc, Il y a une différence significative entre les moyennes des scores des étudiants de l'échantillon au test de la

\section{L'effet du programme:}

Pour connaitre la proportion de l'effet de la variable indépendante sur la variable dépendante, on a calculé la proportion de la valeur d'effet. Elle était 0.88 , donc, elle est significative et élevée.

\begin{tabular}{l|l|l|l|l|l}
\multicolumn{7}{c}{ Tableau N0 } \\
\hline \multirow{2}{*}{ Données } & \multirow{2}{*}{ "t" } & \multirow{2}{*}{ “t” } & Df & \multicolumn{2}{|l}{ El } \\
\cline { 5 - 7 } & & & & $\eta^{2}$ & sig \\
\hline Le test & 11.89 & 141.37 & 20 & 0.88 & élevée \\
\hline
\end{tabular}

$\mathbf{t}=$ valeur de (t) test. $\mathbf{t}^{2}=$ le carré de la valeur de (t) test. $\mathbf{D f}=$ degré de liberté. $\mathbf{s i g}=$ niveau de la signification. $\mathbf{E} \mathbf{l}=$ Effet de l'impact. $\boldsymbol{\eta} \mathbf{2}=$ échelle du carré $($ Itta $)$

Les critères de la mesure de l'impact en termes du carré $\mathrm{du}$ « $\eta^{2} »$ sont: 0.01 ; la petite mesure de l'impact, 0.06; la moyenne mesure de l'impact; 0.14, la grande mesure de l'impact. on trouve que $88 \%$ des changement qui se passent à la variable dépendante (Les compétences de la traduction) reviennent à la variable indépandante (Le programme de la 
recherche). Alors ce programme a un grand effet, donc, il est efficace, car on peut mesurer l'efficacité d'un programme d'après son effet.

\section{L'efficacité du programme:}

Tableau $\mathbf{N}^{0} 5$

\begin{tabular}{l|l|l|l|l}
\hline \multirow{2}{*}{ Données } & \multicolumn{2}{|l|}{ Ma } & $\begin{array}{l}\text { Note } \\
\text { du test }\end{array}$ & Efficacité \\
\cline { 2 - 5 } & Pré & Post & \\
\hline Test & 20.43 & 38.50 & 50 & 0.972 \\
\hline
\end{tabular}

${ }^{73}$ Toutes les opérations statistiques sont élaborées et révisées par le Dr. Taha Ali Ahmed Ali Hassan: professeur de curricula et de méthodologie des maths à la Faculté de Pédagogie, Université de Sohag.

\section{Conclusion}

Au-delà de la pédagogie de l'apprentissage, quelques compétences de la traduction constituent une stratégie convenable qui dépasse le cadre traditionnel utilisé dans l'apprentissage d'une langue étrangère.

L'apprentissage électronique facilite le développement de ces compétences en favorisant une véritable exploration de quelques outils technologiques d'aptitudes déterminés. Ainsi, le e-learning aide les apprenants à contextualiser leur apprentissage concernant l'acquisition de quelques compétences de la traduction. Cette stratégie d'apprentissage crée l'accès pour analyser le texte source, et en produisant le sens original au texte cible.Ce processus d'apprentissage se fait instantanément, soit à la réception du texte à traduire, soit à l'accomplissement de l'activité par l'emploi des outils spécifiques de la traduction. Ainsi, le site du programme intervient comme un médiateur entre les activités traductionnelles. De plus, celui-ci requiert du tuteur, des aptitudes du côté des bases et des règles de la traduction, et se soumet à l'opération de la traduction en ligne dans la phase de la compréhension et de la réexpression.Il est à noter dans cette recherche l'importance de l'apprentissage électronique auquel ont recours les apprenants. Ainsi le processus d'acquisition de nouvelles compétences de la traduction nécessite des connaissances et des aptitudes de la traduction comme suscité, y ajoute un caractère technologique spécial, et une prévoyance supplémentaire.

En terme de conclusion, il est à confirmer que le e-learning met l'accent non seulement sur le savoir mais aussi sur le savoir-faire et le savoirtraduire. La mise en pratique de l'apprentissage électronique dans la classe de FLE est favorable à la traduction du texte. Cette approche réussit à susciter chez les apprenants de $4^{\text {ème }}$ année de la Faculté de Pédagogie de Sohag une réflexion intertextuelle et interculturelle, concernant l'acquisition de quelques compétences de la traduction. 


\section{Références}

Arrouart,C., (2003)."Cadre d'acquisition de compétences en informatique dans la formation universitaire en traduction."Dir. Pierre Auger, co-dir. Denise Deshaies, Université Laval.

Bordeur, J.-P., (2008). Traduction, terminologie, rédaction, vol. 21, n1, 2008, p.17-64.

Buzelin, H., (2002)." Sur le terrain de la traduction ", Thèse de doctorat, Faculté des études supérieures et de la recherche, Université McGill Montréal, Québec.

Eagly, A., \& Chaiken, S. (1993). The psychology of attitudes. Fort Worth; Philadelphia: Harcourt Brace Jovanovich. 364

Forget, N., (1999). "Les dictionnaires électroniques dans l'optique de la traduction." Thèse publiée, École de traduction et d'interprétation, Université d'Ottawa, p.18.

Guidère, M., (2008). La communication multilingue, traduction commerciale et institutionnelle, $1^{\text {ère }}$ édition, de Boeck, Paris. p.5

Page consultée le 6/2/2015

https://books.google.fr/books?isbn $=280415$ 9345

Gouadec, D., (2002). Profession: Traducteur, Paris: La Maison du Dictionnaire.

Honig, H.G., (1991). «Holmes“Mapping Theory" and the landscape of mental translation processes 》, in K.M. van Leuven-Zwart et T. Naaijkens (dir.):
Translation Studies: The State of the Art, Amsterdam/Atlanta, Rodopi, p. 77-89.

Krosnick, J., et al, (2005). The measurement of attitudes. In Johnson, B., \&Zanna, M., (Eds.), The handbook of attitudes (pp. 2122). New York : Psychology Press.

Léchauguette, S., 2015. "Traduire des livres, parcours de formation à la traduction pragmatique pour l'étition ». Thèse de Doctorat, Ecole doctorale Montaigne humanités, Université Michel de Montaigne, Bordeaux III.

Lavault, E., (1985): Fonctions de la traduction en didactique des langues, Didier Erudition, Paris.

Mareschal, G., (2005). Meta, journal des traducteurs / Meta: Translators' Journal, vol. $50, \mathrm{n}^{\circ} 1$, p. 250-262. Page consultée le $3 / 5 / 2015$

http://id.erudit.org/iderudit/010672ar

Nord, C., 2008: La traduction: une activité ciblée. Introduction aux approches fonctionnalistes. Arras: Artois Presses Université.

Rastier, F., (2009). "La traduction: interprétation et genèse du sens », à paraître dans le sens en traduction, Marianne Lederer et Fortunato Israël, éds. Paris, Minard; aussi:

http://www.revuetexto.net/Lettre/Rastier_Traduction.pdf

Solhy, A., (2006). " Un programme suggéré pour développer quelques compétences principales de la traduction chez les professeurs de l'anglais et son effet sur leurs attitudes vers la traduction." thèse de 
Doctorat, méthodologie de la langue anglaise, Faculté de Pédagogie, université de Sud de Vallée de Qéna.

Vinay J.-P, \& Darbelnet, J., (1965):

Stylistique comparée $d u$ français et de l'anglais, PUL. 